\title{
Positron-trapping mechanism at dislocations in $\mathrm{Zn}$
}

\author{
Hidalgo, Carlos; Linderoth, Søren; Diego, Nieves de
}

\section{Published in:}

Physical Review B

Link to article, DOI:

10.1103/PhysRevB.36.6740

Publication date:

1987

\section{Document Version}

Publisher's PDF, also known as Version of record

Link back to DTU Orbit

Citation (APA):

Hidalgo, C., Linderoth, S., \& Diego, N. D. (1987). Positron-trapping mechanism at dislocations in Zn. Physical Review B, 36(13), 6740-6745. https://doi.org/10.1103/PhysRevB.36.6740

\section{General rights}

Copyright and moral rights for the publications made accessible in the public portal are retained by the authors and/or other copyright owners and it is a condition of accessing publications that users recognise and abide by the legal requirements associated with these rights.

- Users may download and print one copy of any publication from the public portal for the purpose of private study or research.

- You may not further distribute the material or use it for any profit-making activity or commercial gain

- You may freely distribute the URL identifying the publication in the public portal

If you believe that this document breaches copyright please contact us providing details, and we will remove access to the work immediately and investigate your claim. 


\title{
Positron-trapping mechanism at dislocations in $\mathrm{Zn}$
}

\author{
Carlos Hidalgo* and Søren Linderoth ${ }^{\dagger}$ \\ Laboratory of Applied Physics II, Technical University of Denmark, DK-2800 Lyngby, Denmark \\ Nieves de Diego \\ Departamento de Fisica de la Materia Condensada, Facultad de Ciencias Fisicas, Universidad Complutense, \\ E-28040 Madrid, Spain
}

(Received 6 March 1987; revised manuscript received 24 June 1987)

\begin{abstract}
The evolution of the positron-lifetime parameters with the measuring temperature has been studied in deformed $\mathrm{Zn}$ in the temperature range $12-210 \mathrm{~K}$. For two samples with different degrees of deformation the presence of a component with a lifetime close to that in a monovacancy is observed. Both the average lifetime and the intensity of the long component decrease with increasing temperature. The experimental results are very well described in terms of a generalized trapping model where it is assumed that positrons become trapped in deep traps (jogs) via shallow traps (dislocation lines). The temperature dependence of the positron-lifetime spectra below $120 \mathrm{~K}$ is attributed to the temperature dependence of the trapping rate to the dislocation line. The experimental results have demonstrated that detrapping processes from the dislocation line take place above $120 \mathrm{~K}$. The positron binding energy to the dislocation line has been shown to be $40 \pm 20 \mathrm{meV}$.
\end{abstract}

\section{INTRODUCTION}

Positron-annihilation spectroscopy (PAS) has proved to be a useful technique in the investigation of defects in many materials, ${ }^{1,2}$ e.g., metals, semiconductors, ionic crystals, and molecular crystals. The problem of positron-dislocation interaction has been undertaken by many investigators since it was first realized that positrons are sensitive to defects introduced by deformation. However, as a consequence of the seeming complexity of the positron-trapping mechanism at dislocations, this problem is still under active discussion.

Positron-lifetime experiments reported for crystals containing dislocations have yielded lifetime values for the trapped positrons close to those of positrons localized at vacancies. One interpretation has been to assume that the lifetime associated with dislocations originates from positrons that annihilate in the dislocation line. By means of this view Arponen et al. ${ }^{3}$ have obtained a positron-dislocation binding energy of about $3 \mathrm{eV}$. For such a binding energy, Bergersen and McMullen ${ }^{4}$ have calculated the temperature dependence of the trapping rate at dislocations, concluding that the trapping rate is temperature independent when the trapping is transition limited, while a temperature dependence results if the trapping is diffusion limited. Martin and Paetsch ${ }^{5}$ treated the positron-dislocation interaction based on pairwise interaction between the positron and metal ions. They concluded that the positron-dislocation binding energy in $\mathrm{Al}$ is very small (less than $0.1 \mathrm{eV}$ ).

An alternative model to describe the positrondislocation interaction has been proposed by Doyama and Cotterill, ${ }^{6}$ who have pointed out that the dislocation line itself might be too "narrow" to localize the positron, while a jog or a vacancy attached to the dislocation core could provide space enough. Smedskjaer et al. ${ }^{7}$ have developed this idea in more detail, studying a mechanism which includes a competition between temperature-dependent detrapping from the dislocation line and trapping to associated point defects with a stronger positron binding energy. Recent experiments dealing with the positron-dislocation interaction have been interpreted by means of this model. ${ }^{8-11}$ In general, the investigation of one kind of defect requires a suitable system which preferably would be a solid containing only the defect under study. This is, of course, advisable for the study of any kind of defect, but probably essential when the problem of the positron-dislocation interaction is considered. The proposed models to describe the interaction are rather complicated, which makes it difficult to obtain unambiguous conclusions when the system is too complex.

Positron-annihilation experiments performed on $\mathrm{Zn}$ irradiated with electrons at low temperatures ${ }^{12}$ did not show formation of three-dimensional vacancy agglomerates when the vacancies migrate in recovery stage III. These results suggest that three-dimensional vacancy agglomerates are not stable in $\mathrm{Zn}$, and as a consequence the deformation of $\mathrm{Zn}$, at temperatures at which vacancies are mobile, should basically produce dislocation-type defects. This fact makes $\mathrm{Zn}$ a suitable system with which to delve deeper into the problem of the positron-dislocation interaction, and with this purpose in mind we have studied deformed $\mathrm{Zn}$ by means of positron-lifetime measurements.

The plan of the present paper is as follows: In Sec. II the experimental details are given; in Sec. III we describe the analysis of the experimental results; in Sec. IV the experimental results are shown; in Sec. $\mathbf{V}$ they are discussed; finally, a brief summary and conclusions are included in Sec. VI. 


\section{EXPERIMENT}

Zinc $(99.9999 \%$ pure) samples were deformed by cold work at $77 \mathrm{~K}$, resulting in a thickness reduction of about $10 \%$. After the deformations the samples labeled $\mathrm{Zn}-\mathrm{I}$ and $\mathrm{Zn}$-II were annealed at room temperature in order to introduce different defect density. Positron-lifetime spectra were obtained by a conventional fast-slow timing spectrometer with a resolution of 246 ps [full width at half maximum (FWHM)]. The specimens were measured at temperatures in the range $12-210 \mathrm{~K}$ with a temperature stability better than $1 \mathrm{~K}$.

As the positron emitter a ${ }^{22} \mathrm{Na}$ source was used, made by evaporating an aqueous ${ }^{22} \mathrm{NaCl}$ solution onto a nickel foil $\left(0.45 \mathrm{mg} \mathrm{cm}^{-2}\right)$. The fraction of positrons annihilating in the nickel foil was estimated ${ }^{13}$ to be about $4 \%$. The component due to annihilations in the salt was obtained from positron-lifetime spectra acquired at room temperature on well-annealed $\mathrm{Zn}$ in which only one component, due to free annihilation in the bulk, is expected. After the foil correction the spectra could be decomposed into two lifetime components: the shorter due to the free annihilations in the bulk $\left(\tau_{f} \simeq 148 \mathrm{ps}\right)$ and the longer $\left(\tau_{2} \simeq 400 \mathrm{ps}\right)$ of faint intensity $(\simeq 3 \%)$ associated with annihilations in the salt. The lifetime spectra were analyzed using the RESOLUTION and POSITRONFIT programs. $^{14}$

\section{METHOD OF ANALYSIS}

Figure 1 is a representation of the positron-trapping model studied in this section. Assume a lattice containing two different kinds of positron traps with concentration $c_{d}$ and $c_{v}$ and corresponding positron lifetimes $\tau_{d}$ and $\tau_{v}$, respectively. The positron-annihilation rates in the perfect lattice and in defects are $\lambda_{f}\left(\equiv 1 / \tau_{f}\right), \lambda_{d}$ $\left(\equiv 1 / \tau_{d}\right)$, and $\lambda_{v}\left(\equiv 1 / \tau_{v}\right)$. Consider a detrapping rate $\epsilon$ for positrons localized at traps labeled $d$ and a transition rate $\eta$ between traps $d$ and $v$. Denoting the specific trapping rate as $v_{i}$, the trapping rate $K_{i}$ can be written as

$$
K_{i}=v_{i} c_{i} \quad(i=d, v) .
$$

Under these circumstances the trapping model shows that the positron-lifetime distribution is a superposition of three exponential components, ${ }^{15}$

$$
n(t)=\sum_{i} I_{i} \exp \left(-\lambda_{i} t\right) \quad(i=\mathrm{I}, \mathrm{II}, \mathrm{III})
$$

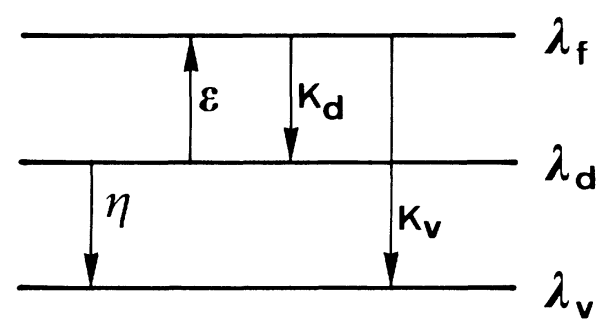

FIG. 1. The trapping model in the presence of two types of defects with possible detrapping from one trap $(d)$ to the perfect lattice or transition to a second trap $(v)$.
However, due to the statistical uncertainties one can only properly decompose the spectra into two lifetime components with the following fitting parameters: $I_{2}$, $\tau_{2}$, and $\tau_{1}$. When it is assumed that the longer-lifetime component, $\tau_{\text {III }}$, is easily separated from the others, the experimentally deduced longer component, $\tau_{2}$, can be identified with $\tau_{\text {III }}$; consequently, $I_{2}$ is identified with $I_{\text {III }}$ and $\tau_{1}$ is the mean lifetime for positrons annihilating with lifetimes $\tau_{\mathrm{I}}$ and $\tau_{\mathrm{II}}$.

From the steady-state condition ${ }^{7}$ the mean positron lifetime can be obtained:

$$
\begin{aligned}
\bar{\tau} & =\tau_{1} I_{1}+\tau_{2} I_{2} \\
& =\frac{\left(\lambda_{d}+\eta+\epsilon\right)\left(1+\tau_{v} K_{v}\right)+K_{d}\left(1+\tau_{v} \eta\right)}{\left(\lambda_{f}+K_{d}+K_{v}\right)\left(\lambda_{d}+\eta+\epsilon\right)-K_{d} \epsilon} .
\end{aligned}
$$

Making use of expression (1), we define an effective trapping rate $K^{*}$ that includes all the physical processes involved in the positron-dislocation interaction:

$$
\begin{aligned}
K^{*} & =K_{v}+\frac{K_{d}}{1+\tau_{d}(\eta+\epsilon)}\left(\frac{\bar{\tau}-\tau_{d}}{\bar{\tau}-\tau_{v}}+\tau_{d} \eta\right) \\
& =\frac{1-\bar{\tau} \lambda_{f}}{\bar{\tau}-\tau_{v}} .
\end{aligned}
$$

In the framework of the general trapping model proposed to describe the positron-dislocation interaction, ${ }^{6,7}$ $\tau_{f} \simeq \tau_{d}$. If we are far from saturation, i.e., $I_{2} \simeq 80 \%$,

$$
\left|\frac{\bar{\tau}-\tau_{d}}{\bar{\tau}-\tau_{v}}\right| \lesssim 1
$$

is verified. Let us consider the particular case when

$$
\eta \tau_{d} \gg 1 \text {. }
$$

Then, positrons initially trapped at the dislocation line escape through two channels: either by diffusing to point defects associated with the dislocation line (i.e., jogs) or by detrapping from the dislocation line back into into the perfect crystal. Under these circumstances expression (2) can by approximated by

$$
K^{*} \simeq K_{v}+K_{d} \frac{\eta}{\eta+\epsilon}=\frac{1-\bar{\tau} \lambda_{f}}{\bar{\tau}-\tau_{v}} .
$$

It should be noticed that with assumption (3) $K^{*}$ becomes the total trapping rate to dislocations in the twotrap model (see Fig. 1).

We wish to stress that by utilizing expression (4) it is possible to relate the temperature dependence of the experimentally deduced parameters $\left(\tau_{2}, I_{2}, \bar{\tau}\right)$ to the temperature behavior of the parameters $\left(K_{d}, K_{v}, \eta, \epsilon\right)$ involved in the trapping model.

\section{RESULTS}

A two-component analysis gave satisfactory fits for the two samples studied, Zn-I and Zn-II, over the entire temperature range studied. The longer positron lifetime was, within the statistical errors, independent of the 
sample temperature, with a value of about 230 ps. Figure 2 shows the results of the free analysis of $\mathrm{Zn}$-I. A final analysis was performed constraining $\tau_{2}$ to $230 \mathrm{ps}$ in order to reduce the statistical scatter. Figure 3 shows the results for $\mathrm{Zn}$-II with $\tau_{2}$ constrained. The reversibility of the curves was always verified.

For the samples Zn-I (Fig. 2) and Zn-II (Fig. 3) three different regions are observed:

Region A: Temperature range $12-80 \mathrm{~K}$; the intensity of the longer component, $I_{2}$, and the mean lifetime, $\bar{\tau}$, are observed to decrease with increasing temperature.

Region B: Temperature range $80-120 \mathrm{~K}$; the mean lifetime, $\bar{\tau}$, and $I_{2}$ are roughly constant.

Region C: Temperature range $120-210 \mathrm{~K}$; the mean lifetime, $\bar{\tau}$, and $I_{2}$ decrease with increasing temperature.

The dashed line shown in Fig. 3 has been deduced from the simple one-trap model. Under this hypothesis the value for the shorter component $\tau_{1}$ is given by

$$
\tau_{1}=\frac{\tau_{f}}{1+K \tau_{f}}
$$

$K$ being the positron-trapping rate to the trap giving rise to the lifetime $\tau_{2}$ :

$$
K=\frac{1-\bar{\tau} \lambda_{f}}{\bar{\tau}-\tau_{2}} .
$$

We notice that the one-trap model fits the experimental results reasonably well. In the same way the agreement with the simple trapping model in the sample $\mathrm{Zn}-\mathrm{I}$ was

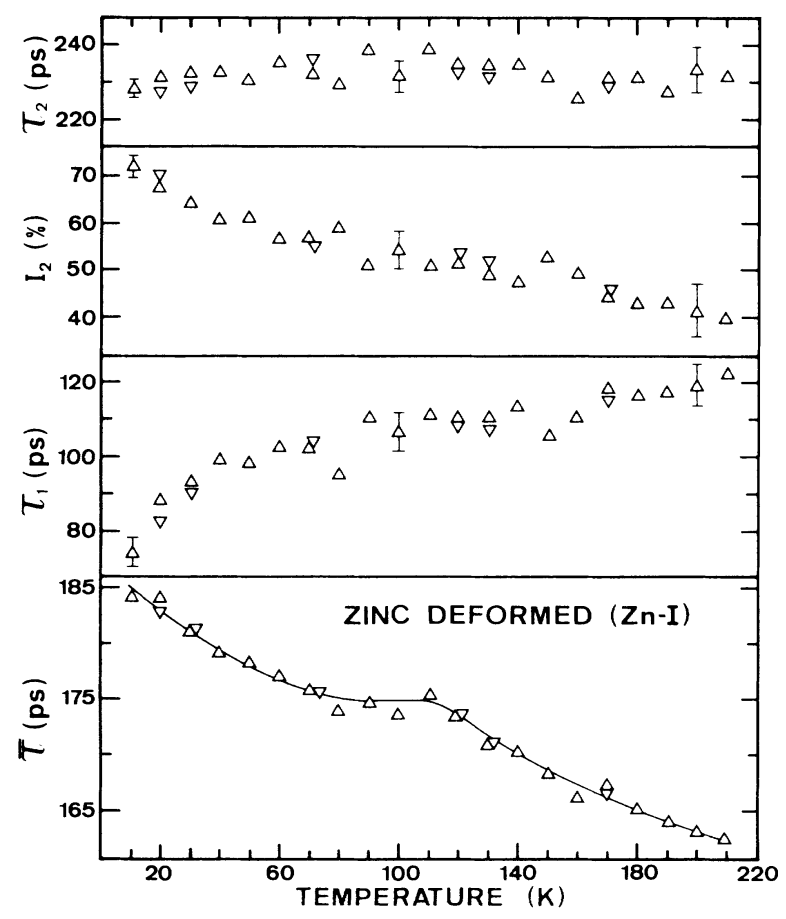

FIG. 2. The positron-annihilation parameters as a function of the measuring temperature for deformed $\mathrm{Zn}$.

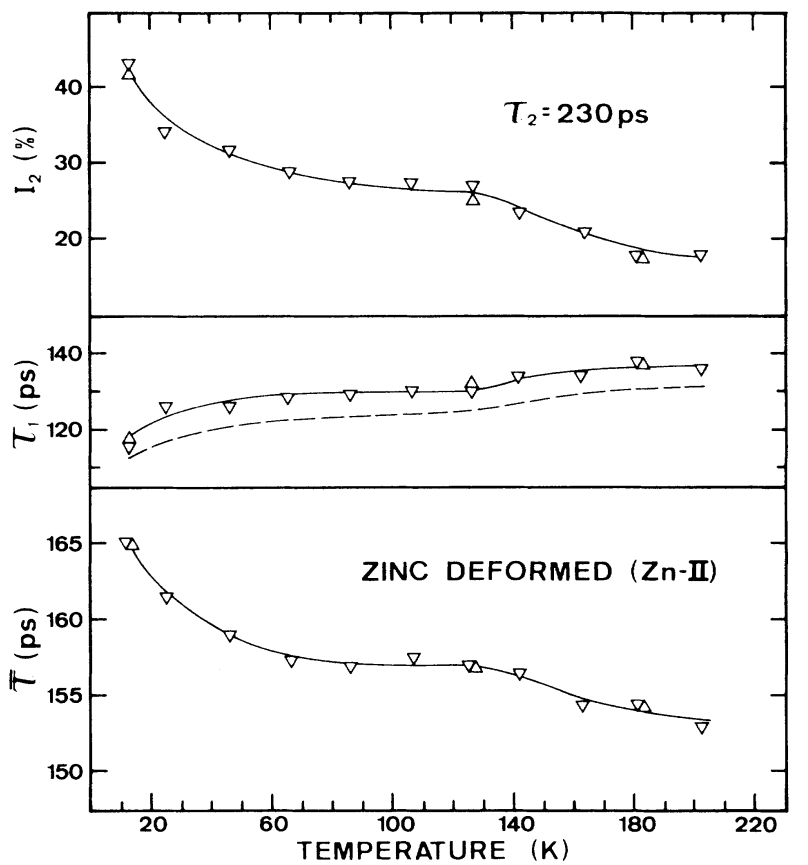

FIG. 3. The positron-annihilation parameters $\tau_{1}, I_{2}$, and $\bar{\tau}$ as a function of the temperature for deformed $\mathrm{Zn}$. The dashed line has been deduced from the one-trap model.

also verified.

The temperature dependence of the effective trapping rate $K^{*}$ is shown in Fig. 4. It has been deduced from expression (4) with $\tau_{2} \equiv \tau_{v}$, taking into account the variation of $\tau_{f}$ with the temperature. In a well-annealed $\mathrm{Zn}$ sample, $\Delta \tau_{f} / \tau_{f}$ has been found experimentally to be $2.6 \times 10^{-2}$ between 77 and $300 \mathrm{~K}$. This temperature dependence was extrapolated to $12 \mathrm{~K}$. Even though this

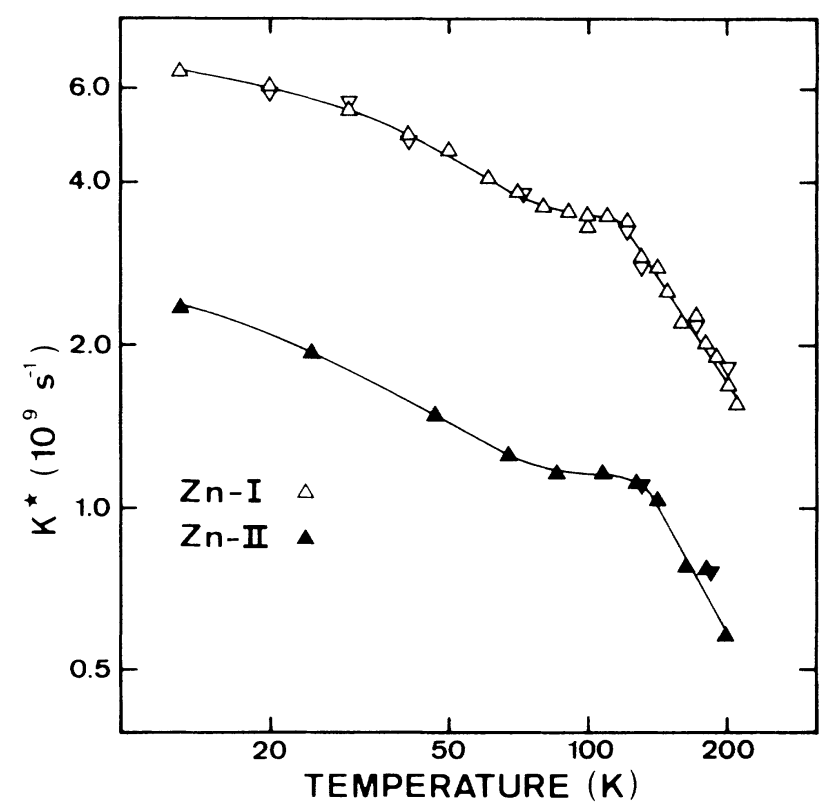

FIG. 4. The effective trapping rate $K^{*}$ as a function of the temperature for $\mathrm{Zn}$ deformed to two different degrees. 
simple extrapolation is a rough approximation, it leads to an insignificant error in the present work. The three different regions previously labeled $\mathrm{A}, \mathrm{B}$, and $\mathrm{C}$ are clearly observed in the temperature dependence of $K^{*}$ for the samples $\mathrm{Zn}-\mathrm{I}$ and $\mathrm{Zn}-\mathrm{II}$.

\section{DISCUSSION}

Considering that the value of the trapping component, $\tau_{2} \simeq 230 \mathrm{ps}$, is very close to the positron lifetime at vacancies, ${ }^{12} \tau=220 \mathrm{ps}$, the existence of three-dimensional vacancy agglomerates can be ruled out in deformed $\mathrm{Zn}$. This conclusion is in agreement with previous results obtained on electron-irradiated $\mathrm{Zn} .{ }^{12}$ The positron lifetime associated with dislocations is, in several metals, observed to be very close to that related to vacancies, ${ }^{6}$ whence we conclude that the positron-trapping component observed in the deformed-Zn samples originates from positron trapping and annihilation at dislocations.

An important question that remains to be answered in the positron-dislocation studies is which trapping model should be utilized to describe the positron-dislocation interaction in $\mathrm{Zn}$. A simple interpretation consists of assuming that the long-lifetime component, $\tau_{2}=230 \mathrm{ps}$, corresponds to positrons trapped and annihilated along the dislocation line (i.e., $K^{*}=K_{d}$ ). In this case, considering the value of the trapping component, it follows that the positron-dislocation-line binding energy should be very close to the positron binding energy at vacancies $\left(E_{b} \simeq 1 \mathrm{eV}\right)$. For such a binding energy the trapping rate at dislocations should be essentially temperature independent when the trapping process is transition limited, while, if the trapping process is diffusion limited, the trapping rate decreases as the temperature increases. ${ }^{4}$

At low temperatures the diffusion constant becomes sufficiently large that the diffusion picture becomes unrealistic; ${ }^{4}$ hence the trapping mechanism should be transition limited and the trapping rate, as previously mentioned, essentially temperature independent. However, Fig. 4 shows no constancy of $K^{*}$ below $120 \mathrm{~K}$, but, on the contrary, a strong temperature dependence is seen. It should also be noticed that in the framework of the diffusion picture the constancy of $K^{*}$ in the temperature range of region $B$ cannot be understood. Hence, we conclude that the positron-dislocation interaction cannot be described assuming that positrons are trapped and annihilated along the dislocation line with a positron binding energy of about $1 \mathrm{eV}$.

An alternative model with which to describe the positron-dislocation interaction, assuming indirect positron trapping into deep traps (jogs and/or vacancies) through shallow traps (dislocation lines) can, on the other hand, easily explain the observed temperature dependence of the effective trapping rate. From the constancy of $\tau_{2}$ with temperature (see Fig. 2), it follows that the longer-lifetime component $\tau_{\text {III }}$ (see Sec. III) is indeed well separated in the experimental spectra. It should be noticed that assumption (3) is experimentally justified since the one-trap model fits the experimental result well; i.e., a negligible fraction of the positrons annihilate in the dislocation line. The effective trapping rate can then be identified with expression (4).

An estimation of the trapping rate directly from the bulk to the deep trap, $K_{v}$, can be made assuming that the specific trapping rate is similar to that at a vacancy. In this case one obtains ${ }^{7}$

$$
K_{v} \simeq \nu_{v} \Omega d a,
$$

where $v_{v}$ is the specific trapping rate at a vacancy $\left(\sim 10^{15} \mathrm{~s}^{-1}\right), \Omega$ the atomic volume, $a$ the number of deep traps per unit dislocation-line length, and $d$ the dislocation density. Several experimental works have monitored the positron-trapping signal as a function of the dislocation density. ${ }^{16-18}$ These works indicate that positron trapping is not observable for dislocation densities less than $10^{8}-10^{9} \mathrm{~cm}^{-2}$. The trapping saturation is found for dislocation densities higher than $10^{11} \mathrm{~cm}^{-2}$. In the present experiments we are not in saturation; hence the dislocation density is estimated to be about $10^{10} \mathrm{~cm}^{-2}$. In such circumstances, even for a density of deep traps as high as $10^{-1} \AA^{-1}$, it is conceivable that $K_{v} \ll K^{*}$. Expression (4) can therefore be approximated by

$$
K^{*} \simeq K_{d} \frac{\eta}{\eta+\epsilon} .
$$

Detrapping processes become less important as the temperature is decreased. Thus let us assume that below $120 \mathrm{~K}$ detrapping processes are negligible (i.e., $\epsilon<\langle\eta$ ). In such circumstances it follows from expression (5) that

$$
K^{*} \simeq K_{d}, \quad T<120 \mathrm{~K} .
$$

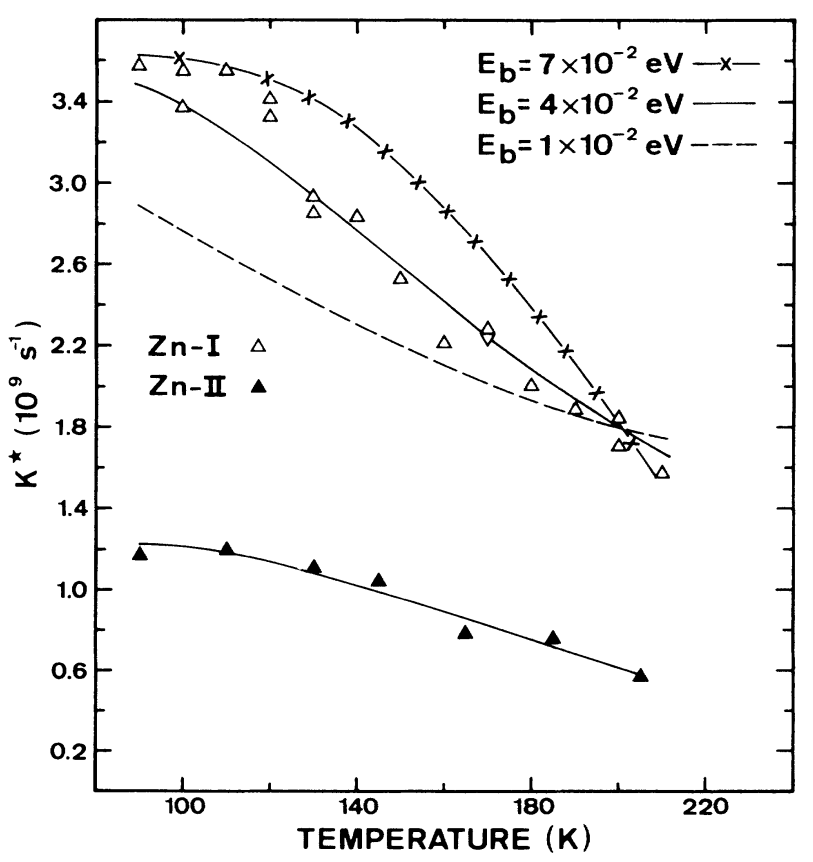

FIG. 5. Fitting of the temperture dependence of the effective positron-trapping rate above $100 \mathrm{~K}$ for three different values of the positron-dislocation binding energy. 
The decrease of the effective trapping rate with increasing temperature in the range $12-80 \mathrm{~K}$ (region $\mathrm{A}$ ) should then be assigned to a decrease of the specific trapping rate, $v$, to the dislocation line with increasing temperature. The temperature dependence of $K^{*}$ (see Fig. 4) found in the range $12-120 \mathrm{~K}$ is consistent with the temperature dependence of $v$ calculated by Smedskjaer et $a .^{7}$ when the positron-dislocation binding energy is close to $10^{-2} \mathrm{eV}$.

If the trapping process is solely diffusion limited the trapping rate is proportional to the positron diffusion constant, ${ }^{15} D_{+}$. Bergersen et al. ${ }^{19}$ have shown that $D_{+} \sim T^{-0.5}$ when the positron diffusion process is limited by positron-acoustic-phonon scattering. Previous measurements performed on fine-grained $\mathrm{Zn}$ alloys ${ }^{20}$ have been shown to be consistent with these calculations. More recently, ${ }^{21}$ measurements carried out on Mo by means of a variable-energy positron beam have confirmed the conclusions of Bergersen et al. Above $120 \mathrm{~K}$ (region $\mathrm{C}$ ) the temperature dependence of the effective trapping rate can be fitted by $K^{*} \sim T^{-\beta}$ ( $\beta \simeq 1.3$ ) for both samples studied; consequently, we conclude that this decrease of $K^{*}$ with increasing temperture cannot be interpreted in terms of the positrondiffusion-limited trapping to the dislocations.

Instead, the decrease of $K^{*}$ above $120 \mathrm{~K}$ (region $\mathrm{C}$ ) can be assigned to positron-detrapping processes from the dislocation line. In this framework the positrondislocation binding energy can be estimated by making use of expression (5). When detrapping processes are active the temperature dependence of $K_{d}$ and $\eta$ should be negligible compared with the temperature dependence of $\epsilon$, which is governed by the exponential ${ }^{15} \exp \left(-E_{b} /\right.$ $\left.k_{B} T\right) . \quad E_{b}$ is the positron-dislocation binding energy, $k_{B}$ the Boltzman constant, and $T$ the temperature. The detrapping rate $\epsilon$ can be calculated as $^{7}$

$$
\epsilon=\left(m v / 4 \pi^{1 / 2} \hbar^{2}\right) E_{b}^{-1 / 2}\left(k_{B} T\right)^{3 / 2} \exp \left(-E_{b} / k_{B} T\right),
$$

where $m$ is the effective positron mass and $v$ the specific trapping rate to the dislocation line. Assuming $K_{d}(T) \simeq K_{d}(100 \mathrm{~K})$ for $T>100 \mathrm{~K}$, and denoting by $T_{s}$ the temperature at which $K^{*}\left(T_{s}\right)=\frac{1}{2} K^{*}(100 \mathrm{~K})$, the temperature dependence of the effective trapping rate $K^{*}$ can be fitted above $100 \mathrm{~K}$ by

$$
\begin{aligned}
K^{*}(T) \simeq & K_{d}(100 \mathrm{~K}) \\
& \times \frac{1}{1+\left(T / T_{s}\right)^{3 / 2} \exp \left(E_{b} / k_{B} T_{s}\right) \exp \left(-E_{b} / k_{B} T\right)} .
\end{aligned}
$$

From Fig. $4, T_{s}$ is estimated to be $200 \pm 10 \mathrm{~K}$ for the two samples investigated. Figure 5 shows the fitting of the experimental results employing expression (7) for three different values of $E_{b}$. The deduced positron-dislocation binding energy is $E_{b}=40 \pm 20 \mathrm{meV}$. This value for $E_{b}$ is in good accord ${ }^{7}$ with the temperature dependence of $K^{*}$ at low temperatures (region A).

\section{CONCLUSIONS}

The positron-lifetime measurements performed on deformed $\mathrm{Zn}$ with different dislocation concentration have led to the following conclusions:

(i) It has been clearly shown that the positrondislocation interaction must necessarily be described by means of a generalized trapping model ${ }^{6,7}$ that includes positron trapping at dislocation lines (shallow traps) from which positrons can either be transferred into the final state (jog and/or vacancy) or be thermally detrapped.

(ii) Below $120 \mathrm{~K}$ the temperature dependence of the effective trapping rate is due to the temperature dependence of the trapping rate into the dislocation line.

(iii) It has been shown that thermally activated positron-detrapping processes from the dislocation line take place above $120 \mathrm{~K}$.

(iv) The positron-dislocation binding energy has been estimated to be around $40 \mathrm{meV}$.

\section{ACKNOWLEDGMENT}

Part of this work was supported by CAICYT (Spain) under Project No. 2016/83.
*Permanent address: Departamento de Fisica de la Materia Condensada, Facultad de Ciencias Fisicas, Universidad Complutense, E-28040 Madrid, Spain.

${ }^{\dagger}$ Present address: Physics Laboratory, H. C. Ørsted Institute, University of Copenhagen, Universitetsparken 5, DK-2100, Denmark.

${ }^{1}$ Positrons in Solids, edited by P. Hautojärvi (Springer-Verlag, Berlin, 1979).

${ }^{2}$ Positron Solid-State Physics, edited by W. Brandt and A. Dupasquier (North-Holland, Amsterdam, 1983).

${ }^{3}$ J. Arponen, P. Hautojärvi, R. Nieminen, and E. Pajanne, J. Phys. F 3, 2092 (1983).

${ }^{4}$ B. Bergersen and T. McMullen, Solid State Commun. 24, 421
(1977).

5J. W. Martin and R. Paetsch, J. Phys. F 2, 997 (1972).

${ }^{6}$ M. Doyama and R. M. J. Cotterill, in Positron Annihilation, edited by R. R. Hasiguti and K. Fujiwara (Japan Institute of Metals, Sendai, 1979), p. 89.

${ }^{7}$ L. C. Smedskjaer, M. Manninen, and M. J. Fluss, J. Phys. F 10, 2237 (1980).

${ }^{8}$ B. Pagh, H. E. Hansen, B. Nielsen, G. Trumpy, and K. Petersen, Appl. Phys. A 33, 255 (1984).

${ }^{9}$ H. Hashimoto, Y. Mosita, and T. Kino, J. Phys. F 15, 1427 (1985).

${ }^{10} \mathrm{C}$. Hidalgo, S. Linderoth, and N. de Diego, Philos, Mag. Lett. A 54, L61 (1986). 
${ }^{11}$ M. D. Bentzon, S. Linderoth, and K. Petersen, in Positron Annihilation, edited by P. C. Jain, R. M. Singru, and K. P. Gopinathan (World-Scientific, Singapore, 1985), p. 485.

${ }^{12}$ C. Hidalgo, N. de Diego, and P. Moser, Appl. Phys. A 40, 25 (1986).

${ }^{13}$ S. Linderoth, H. E. Hansen, B. Nielsen, and K. Petersen, Appl. Phys. A 33, 25 (1984).

${ }^{14}$ P. Kirkegaard, M. Eldrup, O. E. Mogensen, and N. J. Pedersen, Comput. Phys. Commun. 23, 307 (1981).

${ }^{15}$ A. Seeger, Appl. Phys. 4, 183 (1974).

${ }^{16}$ B. T. A. McKee, S. Saimoto, A. T. Stewart, and M. J. Stott,
Can. J. Phys. 52, 759 (1974).

${ }^{17}$ D. Segers, M. Dorikens, and L. Dorikens-Vanpraet, in Positron Annihilation, Ref. 11, p. 564.

${ }^{18}$ G. Dlubek, G. Brauer, O. Brümmer, W. Andreyscheff, and P. Manfrais, Phys. Status Solidi A 30, K37 (1975).

${ }^{19}$ B. Bergersen, E. Pajanne, P. Kubica, M. J. Scott, and C. H. Hodges, Solid State Commun. 15, 1377 (1974).

${ }^{20} \mathrm{C}$. Hidalgo, N. de Diego, and F. Plazaola, Phys. Rev. B 31, 6941 (1985).

${ }^{21}$ H. Huomo, A. Vehanen, M. D. Bentzon, and P. Hautojärvi, Phys. Rev. B 35, 8252 (1987). 